\title{
Targeting HER-2 in gastric cancer - incorporation of trastuzumab into the treatment of operable disease
}

\section{Branislav Bystricky \\ Alicia FC Okines \\ David Cunningham}

Royal Marsden Hospital NHS Trust, Sutton and London, UK
Correspondence: David Cunningham Department of Medicine, Royal Marsden Hospital, Downs Road, Sutton, Surrey, SM2 5PT, UK

Tel +44208 66I 3156

Fax +44 2086613890

Email david.cunningham@rmh.nhs.uk
This article was published in the following Dove Press journal:

Gastrointestinal Cancer:Targets and Therapy

I5 July 2011

Number of times this article has been viewed

\begin{abstract}
Gastric cancer is the fourth most common malignancy and second leading cause of cancer death world-wide, and is therefore a significant global health problem. Radical surgery with a D2 lymph node dissection is an accepted standard approach, and is a key component of multimodality therapy. Perioperative chemotherapy significantly improves 5-year overall survival compared with surgery alone. A significant improvement in overall survival has also been demonstrated with postoperative 5-fluorouracil-based chemoradiotherapy and adjuvant oral S-1 chemotherapy; approaches commonly used widely in North America and Japan, respectively. Approximately $10 \%$ to $20 \%$ of gastric cancers and $20 \%$ to $30 \%$ of esophago-gastric junction cancers are HER-2 positive. The effect of HER-2 overexpression and HER-2 gene amplification on gastric cancer prognosis remains unresolved. The results of the first randomized phase III trial of trastuzumab, a monoclonal antibody directed at the HER-2 receptor, in patients with metastatic gastric cancer were reported recently. Response rate, median progression-free survival, and median overall survival were all significantly improved with the addition of trastuzumab to a cisplatin/fluoropyrimidine doublet. Evaluation of trastuzumab in HER-2 positive operable esophago-gastric cancer is now underway. Lapatinib, a small-molecule inhibitor targeting EGFR and HER-2 is well established in the treatment of trastuzumab-refractory HER-2 positive breast cancer and phase III trials in advanced esophago-gastric cancers are ongoing. Novel, small molecule pan-HER inhibitors have entered early phase evaluation and the antibody-drug conjugate, trastuzumab-DM1, and pertuzumab, a monoclonal antibody which prevents HER-2/ HER-3 dimerization, are currently undergoing phase II/III evaluation in breast cancer. It is our hope that advances in the targeted treatment of HER-2 positive breast cancer will be replicated in HER-2 positive esophago-gastric cancers.
\end{abstract}

Keywords: gastric cancer, adjuvant, perioperative, chemotherapy, trastuzumab, HER-2

\section{Introduction}

Approximately 1 million new cases of gastric cancer were diagnosed in 2008, making gastric cancer the fourth most common malignancy in the world, after lung, breast and colorectal cancers. ${ }^{1}$ The highest incidence is detected in Eastern Asia, Eastern Europe, and South Africa, the lowest in North America. ${ }^{2}$ Regional differences have been attributed to environmental factors, such as high dietary intake of salt, and smoked or cured meats. In addition, there are significant differences in incidence across the socioeconomic groups. Although the incidence and mortality of gastric cancer have been steadily decreasing since 1975, advanced stage at presentation, frequent patient co-morbidities, and the relative chemoresistance of the disease, have all contributed to the poor overall survival (OS). 
The main identified risk factors for gastric cancer are male sex, Helicobacter pylori infection, high levels of dietary salt and nitrates, smoking, medical conditions such as atrophic gastritis and pernicious anemia, previous gastrectomy, and family history. Obesity has been identified as a probable risk factor for gastric cardia and junctional tumors, most likely through increased gastroesophageal reflux and subsequent Barrett's metaplasia. ${ }^{3}$

Sporadic gastric cancers arise through a multi-step process, accumulating mutations in genes regulating key biologic processes, such as those regulating cell cycle, apoptosis, and proliferation. The tumor suppressor gene, $p 53$, plays an important role in cellular response to DNA damage, leading to cell cycle arrest and apoptosis. Mutations in $p 53$ or loss of this gene are found in more than a half of sporadic diffuse and intestinal types of gastric cancer. ${ }^{4}$ Other genes that are mutated or suppressed include CDH1, SMAD4, FHIT, and $C D K N 2 A$, a cyclin-dependent kinase inhibitor. Chromosomal aberrations do not differ between $H$. pylori positive or negative gastric cancers.

The human epidermal growth factor receptor 2 (HER-2) is a transmembrane receptor tyrosine kinase involved in signal transduction and cell growth, encoded by ErbB2 gene. The timing of HER-2 gene amplification in gastric carcinogenesis is uncertain. However, as HER-2 overexpression and amplification is detected in early ${ }^{5}$ as well as in metastatic gastric cancer $^{6}$ and there is high concordance between primary tumors and paired metastatic sites, ${ }^{7}$ this would suggest that it is not a late event.

HER-2 overexpression was first detected in gastric cancer cells in $1986 .{ }^{8}$ In the early 1990s, the role of HER-2 as a potential therapeutic target in gastric cancer was recognized when antibodies blocking HER-2 were reported to inhibit the growth of human gastric tumor cells in vitro, and of established human HER-2 positive tumors in mice xenografts. ${ }^{9}$ However, it was not for another decade when trastuzumab, a humanized monoclonal antibody directed at HER-2, already licensed in breast cancer, was demonstrated to enhance the cytotoxic effects of doxorubicin in gastric cancer cells and to show direct anti-proliferative activity. ${ }^{10,11}$ These and other reports from the preclinical setting precipitated further evaluation of trastuzumab in clinical trials. HER-2 overexpression was found in approximately $20 \%$ of gastric and $33 \%$ of esophago-gastric junction (OGJ) cancers in the screening study for the pivotal ToGA trial. ${ }^{12}$ However, more recent reports have suggested that the rate of HER-2 overexpression may be lower, especially in distal gastric cancers. $^{5}$
This review will focus on trastuzumab and its incorporation into current adjuvant treatment strategies for resectable gastric cancer.

\section{Current strategies for localized gastric cancer \\ Surgery}

Surgery remains critical to the successful treatment of resectable gastric cancer. The optimal extent of lymph node dissection during curative resection for stomach cancer has been a matter of debate for several decades. Japanese surgeons advocate the "D2 resection" comprising en-bloc resection of perigastric nodes and nodes along the main branches of the celiac axis. However, outside Japan, despite radical lymph node dissection, survival remains poor, with 15 -year survival of 33\% for stage II and 19\% for stage IIIA disease reported in a randomized Dutch trial comparing D1 versus D2 lymph node dissection. Although D2 lymphadenectomy was associated with lower loco-regional recurrences $(13 \%$ versus $15 \%$ for D1 group) and lower local and distant recurrences ( $17 \%$ compared with $26 \%$ for D1 group, $P=0.015$ for overall recurrence pattern), a significantly higher surgical mortality and complication rate was observed (10\% and $43 \%$ compared with $4 \%$ and $25 \%$ for D2 and D1 groups, respectively) and no difference in overall survival was detected. ${ }^{13,14}$ Pancreatico-splenectomy was often performed as a part of the D2 lymphadenectomy in this trial, which is likely to have contributed towards the higher surgical mortality. Of note, in the subgroup of patients undergoing D2 resection without pancreatectomy or splenectomy, a significant survival benefit over D1 resection was reported. D2 dissection without resection of the pancreas and spleen is now a standard procedure in patients with resectable tumors. A more extensive lymphadenectomy with para-aortic nodal dissection did not improve overall and recurrence-free survival rates compared with D2 dissection alone. ${ }^{15}$

Several approaches incorporating chemotherapy and radiotherapy into multimodality strategies have been investigated to improve R0 resection and reduce the rate of local and distant recurrences.

\section{Adjuvant chemoradiotherapy}

Adjuvant chemoradiotherapy was investigated by the US Southwest Oncology Group (Intergroup-0116) trial which randomized 556 patients with resected adenocarcinoma of stomach or OGJ to adjuvant chemoradiotherapy or observation only. Patients in the chemoradiotherapy arm received 1 cycle of 5-fluorouracil (5-FU) and leucovorin (LV) 
chemotherapy before, and 2 cycles after chemoradiotherapy with concomitant 5 -FU/LV. ${ }^{16}$ The median survival was 36 months in the chemoradiotherapy arm compared with 27 months in the observation group (hazard ratio [HR] 1.35, $95 \%$ confidence intervals $[\mathrm{CI}]: 1.09-1.66, P=0.005)$ and this benefit was maintained after 10 years of follow-up. ${ }^{16,17}$ Only $10 \%$ of the patients in this study underwent recommended D2 dissection and $54 \%$ had less than D1 dissection, therefore it is unclear whether the radiotherapy is necessary after optimal surgery. Several small, probably underpowered studies have investigated the addition of radiotherapy to adjuvant 5 -FU or cisplatin-based chemotherapy after D2 resection without demonstrating any significant benefit. ${ }^{18,19}$ The role of radiotherapy after optimal surgery will be established by the results of the Korean ARTIST study of adjuvant chemotherapy compared with adjuvant chemoradiation after D2 resection, which are expected shortly. Although postoperative chemoradiotherapy is commonly practiced in US, it has not gained wider acceptance in Europe or Asia.

\section{Perioperative chemotherapy}

Perioperative chemotherapy was investigated by the Medical Research Council Adjuvant Gastric Infusional Chemotherapy (MAGIC) trial in which 503 patients with adenocarcinoma of stomach, lower esophagus, or OGJ were randomized to 3 cycles of chemotherapy with 5-FU, cisplatin and epirubicin (ECF) before and after radical surgery, or to surgery alone. ${ }^{20}$ A significant improvement in 5-year OS (23\% versus 36.3\%, $P=0.009$ for control versus perioperative arms, respectively), higher likelihood of progression-free survival (PFS), higher rate of curative surgery, reduced tumor size, and less advanced nodal disease were reported in the perioperative chemotherapy group. The benefit of perioperative strategy was confirmed by the results of the FNCLCC/ACCORD07/ FFCD 9703 trial, reporting a similar improvement in 5-year survival with perioperative 5 -FU and cisplatin $(\mathrm{CF}) .{ }^{21}$

\section{Adjuvant chemotherapy}

Adjuvant chemotherapy has been investigated in over 30 trials using various agents, either alone or in combination, with the majority reporting no benefit. However, a metaanalysis of individual patient data from 3838 patients in 17 trials reported a benefit from adjuvant chemotherapy compared with surgery alone, with an estimated median OS of 7.8 years following adjuvant chemotherapy compared to 4.9 years following surgery, and absolute survival benefit with adjuvant chemotherapy of $5.8 \%$ at 5 years. ${ }^{22} \mathrm{~A}$ randomized phase III Japanese trial of adjuvant chemotherapy with oral S-1 compared to D2 gastrectomy alone demonstrated significant improvement in OS (from $61 \%$ to $72 \%$ at 5 years) and established this treatment approach as a standard in Japan. ${ }^{23,24}$ Of note, the OS with surgery alone was much higher than reported in studies outside Japan, so that these results cannot currently be extrapolated to a non-Japanese population.

\section{Targeting HER-2 in gastric cancer}

Despite the important advances establishing multimodality treatment for operable gastric cancer, 5-year OS remains poor and new systemic treatment options are urgently needed. Refinement of established adjuvant and perioperative chemotherapy and chemoradiation regimens may confer additional survival benefits, but the magnitude of the gains are likely to be limited. As such, attention has shifted to targeted agents that have known efficacy in other solid tumors. One such target is the HER-2 transmembrane tyrosine kinase receptor, a member of the epidermal growth factor receptor (EGFR) family. The HER-2 receptor is the product of the c-erbB-2/ neu oncogene located on the long arm of chromosome 17, which is involved in signal transduction, cell proliferation, migration, and survival. No ligand has been identified for the HER-2 receptor; instead, the receptor must homodimerize (ligand-independent dimerization) or heterodimerize with another member of the EGFR family, such as HER-3 or HER-4 (ligand-dependent dimerization) to undergo activation, autophosphorylation, and triggering of the downstream signalling cascade. Crystal structure studies of HER-2 receptor have revealed fixed conformation resembling ligandactivated state, capable of interacting with other EGFR family members in the absence of direct ligand binding. ${ }^{25,26}$

HER-2 can enhance signalling by forming a heterodimer with HER-3, where this has been reported to be the most mitogenic dimer. ${ }^{27}$ HER-2 can additionally increase the binding affinity of EGFR or HER-3/4 to their ligands. ${ }^{28,29}$ Binding of the ligand, neuregulin, to HER-3 allows for heterodimerization with HER-2 and subsequent transphosphorylation. Activated HER-3 is capable of activating downstream pathways, and in particular, is a potent activator of phosphatidylinositol-3-kinase (PI3K)/Akt pathways. ${ }^{30}$

HER-2 overexpression or amplification was reported in approximately $20 \%$ of gastric and $33 \%$ of OGJ tumors in patients screened for the ToGA trial. ${ }^{12}$ However, more recent reports suggest that it is a less common finding, especially in distal gastric cancers, where in one study, only $7 \%$ of tumors were reported to be HER-2 positive, compared with $24 \%$ of OGJ cancers. ${ }^{5}$ HER-2 overexpression is more common 
in intestinal type $(32 \%)$ than in diffuse $(6 \%)$ or mixed type gastric cancers $(20 \%)$.

\section{Pharmacology of trastuzumab}

Trastuzumab, a fully humanized monoclonal antibody directed to the extracellular domain of HER-2 receptor was developed in the 1990s after preclinical studies demonstrated an antitumor effect in HER-2 positive breast cancer cell lines. ${ }^{31}$ Trastuzumab binding to the extracellular domain of HER-2 initiates antibody-dependent cell-mediated cytotoxicity, as well as inhibition of downstream HER-2 receptor signalling, leading to cell cycle arrest, reduction of angiogenesis and cell death. ${ }^{30}$

In vitro, trastuzumab inhibits the proliferation of HER-2 amplified gastric cell line NCI-N87 and demonstrates synergistic effect with cisplatin and additive effects with oxaliplatin and 5-FU on the SNU216 gastric cell line. ${ }^{10,32}$ The synergistic effect in combination with cisplatin and capecitabine provided rationale for the phase III ToGA trial. Treatment with trastuzumab produces a significant reduction of HER-2 phosphorylation and reduction in phosphorylated forms of downstream effector molecules such as p-STAT, p-Akt, and p-ERK in these cell lines. In addition, trastuzumab demonstrates antibody-dependent cellular cytotoxicity in the NCI-N87 cell lines. ${ }^{33}$

\section{Trastuzumab in gastric cancer}

In an early report on gastric cancer, HER-2 protein expression, detected by immunohistochemistry (IHC), was positive in $11.9 \%$ of gastric tumors with a negative correlation with survival reported. ${ }^{34}$ However, several other studies have explored the prognostic impact of HER-2 overexpression or amplification, with conflicting results. The largest, a combined study from the UK and Germany of 924 resected gastric cancer specimens was unable to determine any prognostic value of HER-2, and less than $10 \%$ of cases were reported to be HER-2 positive. ${ }^{35}$ These conflicting results most likely reflect the use of nonstandardized assays, intratumoral heterogeneity of the HER-2 staining pattern and variation in tissue processing with subsequent differences in the HER-2 scoring between these studies. A standardized scoring system for gastric cancer was developed and validated for the ToGA trial and has now been accepted into routine clinical practice. ${ }^{36,37}$ The recommended scoring for HER-2 in advanced gastric cancer is summarized in Table 1. The notable differences between gastric and breast cancer HER-2 scoring relate to an increased frequency of incomplete membranous staining and intratumoral heterogeneity of HER-2 expression in gastric cancer. ${ }^{36}$

In the pivotal randomized phase III ToGA trial of trastuzumab in gastric cancer, HER-2 status was evaluated in 3807 patients by IHC and fluorescence in situ hybridization (FISH). HER-2 positivity was defined as either IHC 3+ or FISH positive (equal or more than 2 HER-2 gene signals to centromere 17 signals, HER2/CEN17 $\geq 2$ ) in patients with IHC $0,1+$ or $2+$. Patients with IHC 0 and $1+$ disease were eligible if the cancer was FISH+, which is in contrast to guidelines from American Society of Clinical Oncology (ASCO) for breast cancer (IHC 3, IHC 2+ with FISH +, HER2/CEN17 $\geq 2.2) .{ }^{38}$ Five

Table I The recommended scoring for HER-2 in advanced gastric cancer

\begin{tabular}{|c|c|c|c|}
\hline \multicolumn{4}{|l|}{ Evaluation criterion } \\
\hline \multirow[t]{2}{*}{ Number of cells considered positive } & \multicolumn{3}{|c|}{5 cells in biopsy } \\
\hline & \multicolumn{3}{|c|}{$10 \%$ in surgical specimen } \\
\hline \multirow[t]{2}{*}{ Pattern of membrane staining } & \multicolumn{3}{|c|}{ Distinct and regular } \\
\hline & \multicolumn{3}{|c|}{ Often lateral or U-shaped reactivity } \\
\hline \multirow[t]{3}{*}{ Score assignment } & Negative & $0-I+$ & No/faint reactivity, only in part of membrane \\
\hline & Equivocal & $2+$ & Weak/moderate, complete/basolateral reactivity \\
\hline & Positive & $3+$ & $\begin{array}{l}\text { Moderate/strong, complete/basolateral reactivity, } \\
\text { visible at low magnification }\end{array}$ \\
\hline \multirow[t]{5}{*}{ Histologic tumor type/site } & \multicolumn{3}{|c|}{$32 \%$ interstitial type } \\
\hline & \multicolumn{3}{|c|}{$20 \%$ mixed type } \\
\hline & \multicolumn{3}{|c|}{$6 \%$ diffuse type (signet-ring usually negative) } \\
\hline & \multicolumn{3}{|c|}{$20 \%$ gastric origin } \\
\hline & \multicolumn{3}{|c|}{$33 \%$ OGJ } \\
\hline Heterogeneity & \multicolumn{3}{|c|}{ Frequent within the same tumor } \\
\hline \multirow[t]{3}{*}{ In situ hybridization } & \multicolumn{3}{|c|}{ FISH - most widely used in reported studies } \\
\hline & \multicolumn{3}{|c|}{$\mathrm{CISH}, \mathrm{SISH}, \mathrm{BDISH}$ - allow for morphologic evaluation in addition to gene amplification } \\
\hline & \multicolumn{3}{|c|}{ HER $2 /$ CEN 17 ratio $\geq 2$} \\
\hline
\end{tabular}

Abbreviations: OG], esophagogastric junction; FISH, fluorescence in situ hybridization; $\mathrm{CISH}$, chromogenic in situ hybridization; SISH, silver in situ hybridization; BDISH, brightfield double in situ hybridization. 
hundred and eighty-four eligible patients were randomized to receive cisplatin $80 \mathrm{mg} / \mathrm{m}^{2}$ iv on day 1 with fluoropyrimidine (capecitabine $1000 \mathrm{mg} / \mathrm{m}^{2}$ orally twice daily for 14 days or 5 -FU $800 \mathrm{mg} / \mathrm{m}^{2}$ on days 1-5) with or without trastuzumab on a 3-weekly schedule. Patients received up to 6 cycles of chemotherapy with or without trastuzumab, and those randomized to the investigational arm continued to receive the antibody until disease progression. Median OS was 13.8 months in the trastuzumab arm compared to 11.1 months in the chemotherapy only arm (HR 0.74, 95\% CI 0.60-0.91, $P=0.0046$ ). Response rate and median PFS were also increased (5.5 to 6.7 months, HR $0.71,95 \%$ CI $0.59-0.85, P=0.0002) .{ }^{6}$ In a preplanned analysis by HER-2 expression level, patients with IHC3+ disease attained the greatest benefit from the addition of trastuzumab, with a median survival of 17.9 vs 12.3 months (HR 0.58, 95\% CI 0.41-0.81). Furthermore, in an exploratory subgroup analysis of the ToGA trial which excluded the IHC $0-1+$ patients in line with breast cancer convention, the magnitude of benefit was again greater than for the intention to treat population, and median OS was increased from 11.8 months to 16.0 months with the addition of trastuzumab (HR 0.65 , 95\% CI 0.51-0.83). These results are summarized in Table 2. Despite these results being derived from a post-hoc exploratory analysis, the European Medicine Agency (EMA) has limited the approval of trastuzumab to patients with IHC $3+$ or $2+/$ $\mathrm{FISH}+$ metastatic gastric/OGJ adenocarcinoma. Moreover, the UK National Institute for Clinical Excellence has restricted its recommendation for trastuzumab to patients with IHC3+ disease only, based upon a cost-efficacy analysis. The FDA has approved trastuzumab for HER-2 overexpressing patients without further specification.
Trastuzumab is well tolerated both as monotherapy and in combination with several regimens in breast cancer studies. ${ }^{39-44}$ The only significant overlapping toxicity identified is cardiotoxicity, where trastuzumab cannot be safely delivered concurrently with standard cumulative doses of the anthracycline, doxorubicin: the incidence of cardiac dysfunction was reported in up to $27 \%$ of patients..$^{42}$ Instead, sequential delivery is feasible, with more acceptable rates of cardiotoxicity reported. ${ }^{39-41,43,44}$ The combination of trastuzumab and anthracyclines has been investigated in neoadjuvant and metastatic setting in breast cancer. Trastuzumab in combination with neoadjuvant FEC regimen utilizing lower epirubicin dose of $75 \mathrm{mg} / \mathrm{m}^{2}$ for 4 cycles was well tolerated in 23 operable patients. No cases of congestive heart failure (CHF) were observed and although an asymptomatic drop in left ventricular ejection fraction (LVEF) was noted in 7 patients, there was return to normal levels on follow-up evaluation. ${ }^{45}$ Similarly, 4 cycles of epirubicin $\left(90 \mathrm{mg} / \mathrm{m}^{2}\right)$ with cyclophosphamide were safely delivered in combination with trastuzumab in neoadjuvant GeparQuattro study. Trastuzumab was given in $78 \%$ of 445 HER-2 positive patients, and each $\mathrm{CHF}$, cardiac ischemia and persistent decrease in LVEF occurred only in 1 patient. $^{46}$ Six cycles of the same regimen were used in HERCULES trial which randomized 120 patients into 2 different doses of epirubicin $\left(60\right.$ and $\left.90 \mathrm{mg} / \mathrm{m}^{2}\right)$ in combination with cyclophosphamide and trastuzumab. Asymptomatic LVEF declines and symptomatic CHF were more frequent with the higher dose of epirubicin, but all $\mathrm{CHF}$ occurred after the end of chemotherapy administration and resolved or improved with or without specific treatment. ${ }^{47}$ Trastuzumab delivered concurrently with doxorubicin has

Table 2 Overall, preplanned, and post-hoc exploratory analyses of patients in the ToGA trial'2 stratified by HER-2 status

\begin{tabular}{|c|c|c|c|c|c|c|}
\hline & & \multirow[t]{2}{*}{$\mathbf{n}$} & \multicolumn{2}{|l|}{ Median OS } & \multirow[t]{2}{*}{ HR } & \multirow[t]{2}{*}{$95 \% \mathrm{Cl}$} \\
\hline & & & $\begin{array}{l}\text { Trastuzumab + } \\
\text { chemotherapy }\end{array}$ & $\begin{array}{l}\text { Chemotherapy } \\
\text { only }\end{array}$ & & \\
\hline \multicolumn{7}{|c|}{ Overall population } \\
\hline $\mathrm{FISH}+$ or IHC $3+$ & & 584 & 13.8 & II.I & 0.74 & $0.6-0.91$ \\
\hline \multicolumn{7}{|c|}{ Pre-planned exploratory analysis } \\
\hline \multirow[t]{4}{*}{$\mathrm{FISH}+$} & $\mathrm{IHC} 0$ & 61 & 10.6 & 7.2 & 0.92 & $0.48-1.76$ \\
\hline & $\mathrm{IHC} \mathrm{I+}$ & 70 & 8.7 & 10.2 & 1.24 & $0.7-2.2$ \\
\hline & IHC 2+ & 159 & 12.3 & 10.8 & 0.75 & $0.5 \mathrm{I}-\mathrm{I} . \mathrm{II}$ \\
\hline & $\mathrm{IHC} 3+$ & 256 & 17.9 & 12.3 & 0.58 & $0.4 I-0.8 I$ \\
\hline FISH- & $\mathrm{IHC} 3+$ & 15 & 17.5 & 17.7 & 0.83 & $0.2-3.38$ \\
\hline \multicolumn{7}{|c|}{ Post-hoc exploratory analysis } \\
\hline $\mathrm{FISH}+$ & $\mathrm{IHC} 0, \mathrm{I}+$ & $|3|$ & 10.0 & 8.7 & 1.07 & $0.7-1.62$ \\
\hline $\begin{array}{l}\mathrm{FISH}+ \\
\text { or } \mathrm{IHC} 3+\end{array}$ & $\mathrm{IHC} 2+$ & 446 & 16.0 & 11.8 & 0.65 & $0.5 \mathrm{I}-0.83$ \\
\hline
\end{tabular}

Abbreviations: OS, overall survival; FISH, fluorescence in situ hybridization; IHC, immunohistochemistry; $\mathrm{HR}$, hazard ratio; $\mathrm{Cl}$, confidence intervals. 
also been re-evaluated in Neoadjuvant Herceptin (NOAH) trial. Doxorubicin was given at $60 \mathrm{mg} / \mathrm{m}^{2}$ for 3 cycles in a combination regimen with or without trastuzumab. CHF was observed in 2 patients treated with trastuzumab (2\%) compared with 0 in the chemotherapy only arm. ${ }^{48}$

In the ToGA trial, the adverse event profile was similar between the 2 groups, with no difference in the overall rate of adverse events. The frequency of CHF was less than $1 \%$ in both arms; and asymptomatic drops in LVEF (defined as $\geq 10 \%$ and to $<50 \%$ ) was $1 \%$ compared with $5 \%$ in the control and experimental arms, respectively. The frequency of CHF and ventricular dysfunction may have been confounded by relatively short follow-up in this study due to poor OS of metastatic gastric cancer, but importantly, the rate of symptomatic events which could affect quality of life (QoL) was low.

\section{Impact of trastuzumab on quality of life}

QoL is a perception of one's health, and includes physical, emotional and social function in addition to disease and treatment-related side-effects. ${ }^{49}$ Assessment of quality of life by validated questionnaires (European Organisation for Research and Treatment of Cancer (EORTC) QLQ-C30) as well as gastric-cancer specific module STO22 were used in the ToGA trial. Global health status scores from the QLQC30 increased during the chemotherapy and showed further improvements after chemotherapy was ceased in both arms. Physical, emotional, and social functions improved after chemotherapy and cognitive functions remained same in both arms. Disease-specific scores from the QLQ-ST022 (evaluating dysphagia, anxiety, reflux, and eating restrictions) decreased after the first cycle of chemotherapy, indicating improvement in disease-specific symptoms. ${ }^{50}$ Overall, the addition of trastuzumab did not negatively impact in the QoL of participants in the ToGA trial.

\section{Trastuzumab in the adjuvant treatment of gastric cancer}

The benefit of trastuzumab in the post-hoc analysis of the ToGA trial in the treatment of advanced gastric cancer approximates that reported in the treatment of advanced breast cancer. Absolute increases in overall survival of 4 and 5 months were reported in the ToGA trial and the first phase III advanced breast cancer trial, respectively. ${ }^{6,42}$ Trastuzumab also improves survival in the adjuvant treatment of HER-2 positive breast cancer. ${ }^{40,41,51}$ However, activity of targeted agents in metastatic disease should not automatically be extrapolated into adjuvant setting, as several trials with active agents in metastatic colon cancer were negative in the treatment of early disease. ${ }^{52-54}$ Evaluation of trastuzumab in the perioperative treatment of gastric cancer is therefore critical to determine if a similar improvement in survival can be gained. As yet, only 2 trials recently open to recruitment are investigating HER-2 targeting in this setting. A small phase II study (ML25189) plans to accrue 45 patients with resectable, HER-2 positive adenocarcinoma of stomach or OGJ type I-III into a single treatment arm consisting of 3 cycles of neoadjuvant chemotherapy with oxaliplatin, capecitabine and trastuzumab. Patients achieving R0 or R1 resection will receive a further 3 cycles of the same chemotherapy regimen postoperatively with trastuzumab continuing for 12 months. The primary endpoint of this trial is DFS at 18 months. ${ }^{55}$ A larger, phase III trial sponsored by US Radiation Therapy Oncology Group (RTOG)-1010 plans to enrol 480 patients with HER-2 positive adenocarcinoma of middle or lower esophagus or OGJ. The extent of the disease must be encompassed in one radiotherapy field and patients must be able to undergo curative resection after completion of chemoradiotherapy treatment. Eligible patients are randomized to chemotherapy with weekly paclitaxel and carboplatin for 6 weeks with concomitant radiotherapy. The experimental arm consists of the same chemotherapy schedule with the addition of weekly trastuzumab and an additional trastuzumab dose on day 57. Commencing 3 to 14 weeks post resection, all patients receive 13 cycles of 3 -weekly trastuzumab. The primary endpoint is DFS and secondary outcomes are pathologic complete response (pCR), OS, toxicity, QoL, predictors of cardiotoxicity and molecular correlates of efficacy. ${ }^{56}$ These studies are summarized in Table 3.

\section{Other HER-2 targeting agents}

Pertuzumab is a monoclonal antibody which inhibits HER-2 heterodimerization. Its binding site on HER-2 receptor is different from trastuzumab, therefore these compounds do not compete with one another. Pertuzumab delivered in combination with trastuzumab has been evaluated in a phase II study in patients with trastuzumab-resistant HER-2 positive metastatic breast cancer. The median PFS reported was 5.5 months and the objective response rate of $24 \%$ was encouraging in this pretreated patient population. ${ }^{57}$ Pertuzumab has also been evaluated in a randomized comparison of gemcitabine plus pertuzumab or placebo in a HER-2-unselected population of patients with platinum-resistant ovarian cancer. A significant increase in response rate was reported, with greatest benefit noted in the subgroup of patients with low HER-3 mRNA expression. ${ }^{58}$ 
Table 3 Active, recruiting phase II/III studies of neoadjuvant and adjuvant trastuzumab in gastric or esophageal cancer

\begin{tabular}{|c|c|c|c|c|c|c|}
\hline $\begin{array}{l}\text { Study and sponsor, } \\
\text { Clinical trials.gov ID }\end{array}$ & $\mathbf{n}$ & Phase & Inclusion & Schedule & $\begin{array}{l}\text { Primary } \\
\text { endpoint }\end{array}$ & Country \\
\hline $\begin{array}{l}\text { ML25I } 89 \\
\text { Hoffmann-La Roche, } \\
\text { NCT0I I } 30337^{55}\end{array}$ & 45 & II & Gastric adenocarcinoma & $\begin{array}{l}3 \times \text { CAPOX }+\mathrm{H} \text { neoadjuvant } \\
3 \times \text { CAPOX }+\mathrm{H} \text { adjuvant } \\
12 \text { months adjuvant trastuzumab }\end{array}$ & DFS & Spain \\
\hline $\begin{array}{l}\text { RTOG-I0I0 } \\
\text { NCI/RTOG, } \\
\text { NCTOII } 96390^{56}\end{array}$ & 480 & III & $\begin{array}{l}\text { Middle, lower esophageal } \\
\text { and OGJ adenocarcinoma }\end{array}$ & $\begin{array}{l}\text { Weekly carboplatin, paclitaxel and } \\
\text { radiotherapy with or without } \mathrm{H} \\
(6 \text { weeks })+\text { additional dose of } \mathrm{H} \\
\text { on day } 57 \text {. Surgery within } 5-6 \text { weeks } \\
\text { after completion of radiotherapy; } \\
\text { adjuvant } \mathrm{H} \text { for } 39 \text { weeks for all } \\
\text { patients }\end{array}$ & DFS & USA \\
\hline
\end{tabular}

Abbreviations: CAPOX, capecitabine and oxaliplatin; H, trastuzumab; DFS, disease-free survival; NCl, National Cancer Institute; RTOG, Radiation Therapy Oncology Group; OGJ, esophagogastric junction.

Several phase III trials are currently investigating pertuzumab in trastuzumab-naïve as well as trastuzumab-refractory patients with breast cancer.

Trastuzumab emtansine (trastuzumab-DM1) is a conjugate of cytotoxic drug maytansine derivative DM1 and trastuzumab. DM1 binds microtubules, inhibits their assembly and blocks mitosis, similarly to vinca alkaloids. By conjugating DM1 to trastuzumab, the delivery of DM1 is more specific and reduces its systemic toxicity. A single arm phase II study demonstrated a response rate of $26 \%$ and median PFS of 4.6 months in the treatment of breast cancer patients who were refractory to prior HER-2 targeted therapies. ${ }^{59}$ Two large phase III trials evaluating the addition of trastuzumab-DM1 to lapatinib and capecitabine in pretreated patients, and in combination with pertuzumab compared with trastuzumab plus a taxane in the first-line setting in advanced breast cancer, are currently open to recruitment. ${ }^{60,61}$ Trastuzumab-DM1 has antitumor activity in HER-2 positive gastric cell lines and xenografts, as well as in trastuzumab-resistant tumors. ${ }^{62}$ These encouraging preclinical data provide rationale for clinical trials of trastuzumab-DM1 in trastuzumab-resistant gastric cancer, and might represent a useful second-line treatment option, potentially free of the sideeffects associated with systemic cytotoxic chemotherapy.

Lapatinib, a small molecule inhibitor targeted at the intracellular tyrosine-kinase domains of HER-2 and EGFR has been investigated extensively in trastuzumab-refractory breast cancer. In the pivotal phase III study, heavily pretreated HER-2 positive patients were randomized to oral capecitabine chemotherapy with or without lapatinib. Response rate and median time to progression were significantly increased with the addition of lapatinib and the combination was well tolerated. Diarrhea was the most common grade 3-4 toxicity, reported in $14 \%$ with the combination compared to $11 \%$ with capecitabine alone. ${ }^{63}$ There were no symptomatic cardiac events reported and LVEF values were comparable at scheduled assessments, with 4 asymptomatic cardiac events noted in the combination group, compared with 1 in the monotherapy arm.

Lapatinib has been investigated in a phase II, single arm study in the first-line treatment of advanced gastric cancer. Overall response rate was $9 \%$ and median OS 5 months. ${ }^{64}$ Even though lapatinib has demonstrated modest single-agent activity, the response rates and survival were poor in this study compared with cytotoxic chemotherapy in first-line treatment of gastric cancer, therefore combination studies are underway. Two large randomized phase III studies in advanced gastric cancer are currently accruing patients; the LOGiC study will evaluate the combination of lapatinib with oxaliplatin and capecitabine as first-line treatment and the TYTAN study will evaluate lapatinib in combination with weekly paclitaxel in the second-line setting. ${ }^{65,66}$ No significant safety issues were reported on interim analyses of these studies. ${ }^{67,68}$ An amendment to the UK National Cancer Research Institute phase III study of neoadjuvant chemotherapy ECX with or without bevacizumab (ST03) is planned, to introduce baseline HER-2 testing and randomization of HER-2 positive patients to ECX with or without lapatinib.

Second-generation, irreversible pan-HER tyrosine kinase inhibitors are currently being intensely investigated in early phase clinical trials. PF-00299804 has demonstrated significant antitumor activity in human xenograft models, including xenografts with activating mutations in EGFR that are associated with resistance to the anti-EGFR tyrosine kinase inhibitors, gefitinib and erlotinib. ${ }^{69} \mathrm{~A}$ phase II trial in advanced nonsmall cell lung cancer has reported efficacy in erlotinib-pretreated patients. ${ }^{70} \mathrm{~A}$ phase II monotherapy study in advanced gastric cancer is underway. ${ }^{71}$ Neratinib, a further irreversible pan-HER inhibitor has demonstrated efficacy in combination with trastuzumab and as monotherapy in trastuzumab-refractory breast 


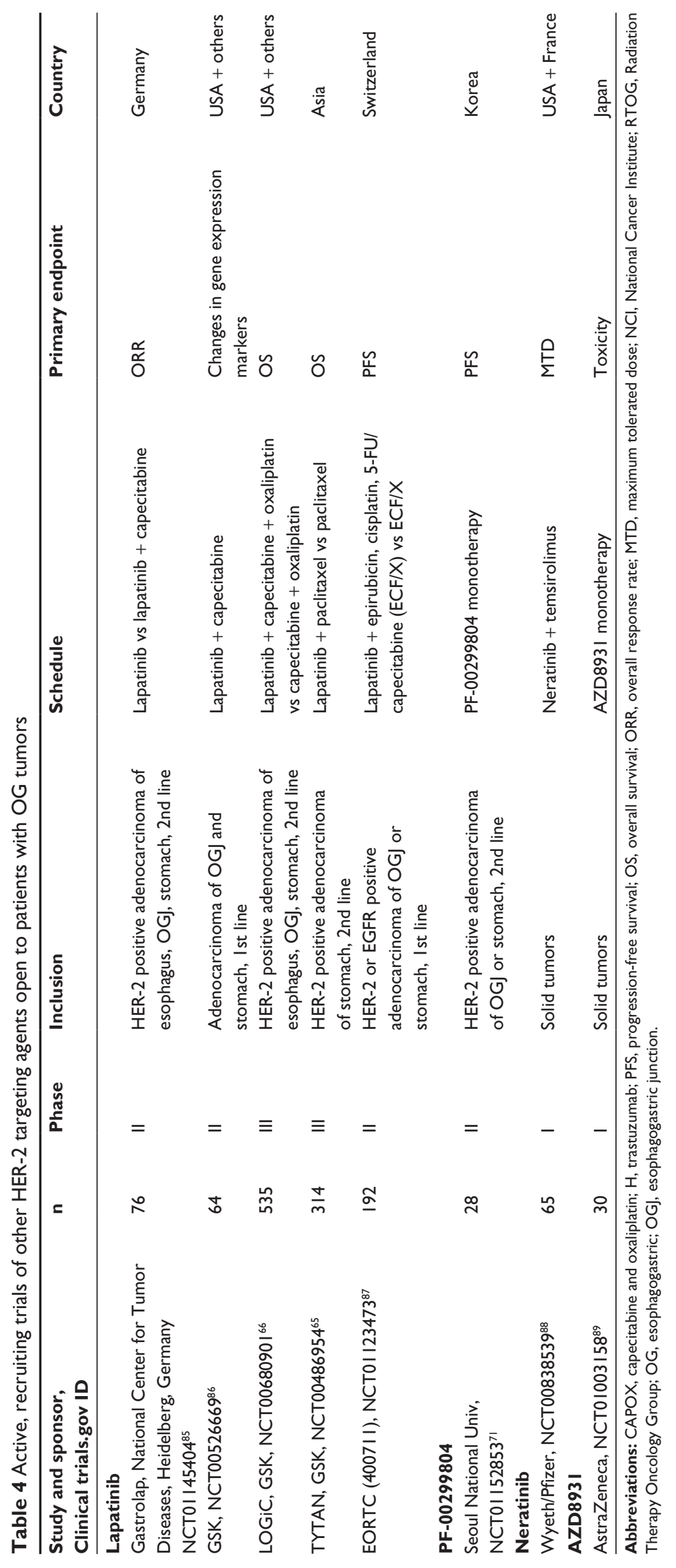


cancer patients. ${ }^{72}$ Anti-tumor activity of AZD8931, a reversible pan-HER inhibitor was confirmed in preclinical models, significantly affecting EGFR, erbB2, and erbB3 phospohorylation and downstream signalling pathways. ${ }^{73}$ Early phase clinical trials are currently ongoing in breast cancer and other solid tumors. We remain hopeful that these new agents will be further investigated in patients with esophagogastric tumors in future clinical trials. Current ongoing clinical trials with these agents that are recruiting patients with gastro-intestinal and/or solid tumors are summarized in Table 4.

\section{Future directions}

In addition to the currently planned evaluation of trastuzumab in the perioperative and adjuvant settings and of lapatinib in the perioperative setting, further important questions on the role of HER-2 targeting in gastric cancer remain. The safety of trastuzumab in combination with the widely-used ECF or ECX regimens has not yet been established. Neo-adjuvant studies in breast cancer ${ }^{45,46,48,74}$ support the concurrent use of trastuzumab with anthracyclines, provided that less cardiotoxic anthracyclines, such as epirubicin, and low cumulative doses are used, with careful cardiac monitoring and early cessation of the cardiotoxic agents where indicated. The cumulative epirubicin dose in the MAGIC trial of $300 \mathrm{mg} / \mathrm{m}^{2}$ is well below the maximum recommended cumulative dose of $900 \mathrm{mg} / \mathrm{m}^{2} .^{75}$ Trastuzumab should therefore be evaluated with perioperative ECF or ECX for carefully selected patients according to pre-existing cardiac conditions and baseline LVEF, with careful monitoring for cardiotoxicity. However, to our knowledge, no such study is currently planned.

The optimal duration of trastuzumab in the adjuvant treatment of breast cancer has not yet been established, with benefits reported from as little as 9 weeks treatment. ${ }^{39}$ One year's adjuvant therapy is the currently accepted standard, based upon the survival benefit reported in the NSABP B-31, BCIRG 006, and HERA studies. ${ }^{40,41,44}$ Whether continuation for longer than 1 year is beneficial is not currently known.

Delivery of trastuzumab beyond disease progression has been widely practiced in advanced breast cancer treatment and should be prospectively evaluated in advanced gastric cancer as soon as an effective second-line treatment is established. Response rates as well as median time to progression were improved in HER-2 positive metastatic breast cancer patients who experienced progression during trastuzumab treatment and were randomized to capecitabine with continuation of trastuzumab, compared with capecitabine alone. ${ }^{76}$ Several phase II trials have investigated second-line chemotherapy in esophago-gastric cancers using irinotecan and taxanes, ${ }^{77-79}$ but phase III data are currently lacking. Trials evaluating trastuzumab beyond progression, and other HER-2 targeting agents in trastuzumab pretreated patients with esophago-gastric cancers, are urgently needed.

The combination of trastuzumab and lapatinib is synergistic in breast cancer, therefore evaluation in advanced and, in the future, localized gastric cancer is warranted. Preclinical studies have demonstrated synergistic effect of lapatinib and trastuzumab in gastric cancer cell lines; synergy was also demonstrated in HER-2 amplified xenografts. ${ }^{80}$ These preclinical data provide rationale for combination studies of these agents in gastric cancer.

The combination of trastuzumab with pertuzumab may also be worthy of investigation in early gastric cancer. Activity of pertuzumab and trastuzumab demonstrated in trastuzumab-resistant breast cancer has precipitated ongoing first-line and neoadjuvant studies in breast cancer. Extended HER-2 blockade with enhanced antitumor efficacy makes this combination an attractive investigational therapy in esophagogastric cancers and should be evaluated without delay.

Compensatory signalling and cross-talk between HER-2 and other family members is likely to contribute to trastuzumab resistance. Blocking multiple HER homo- and heterodimers have demonstrated enhanced growth inhibition when compared with single agents. ${ }^{81}$ Highly-selective, small molecule pan-HER inhibitors have the potential to improve antitumor activity and overcome trastuzumab resistance. Encouraging results have already been reported in nonsmall cell lung cancer. ${ }^{82}$ The relative merit of the pan-HER inhibitors compared with more selective antiHER-2 targeting will be of interest, and will determine the future role of these agents in breast and gastric cancers.

Strategies to overcome primary and acquired resistance to trastuzumab are an area of intense investigation in breast cancer oncology. Combination with PI3K inhibitors and mTOR inhibitors are currently undergoing evaluation. Rapid investigation into mechanisms of resistance in gastric cancer is needed to determine whether the same or different mechanisms predominate, to establish whether successful strategies in breast cancer can similarly be employed in trastuzumab-resistant gastric cancer. Irreversible pan-HER inhibitors could abrogate some of the mechanisms of trastuzumab resistance, and new dual PI3K and mTOR inhibitors are currently under investigation this setting. ${ }^{83,84}$

\section{Conclusion}

Multimodality therapy with surgery, plus adjuvant fluoropyrimidine-based chemotherapy or chemoradiation or perioperative combination chemotherapy, are the current 
standard treatment options for operable gastric cancer. However, survival remains poor and new strategies incorporating targeted agents are needed.

In advanced gastric and OGJ cancer, establishing the HER-2 status has become part of the routine diagnostic histopathological assessment and trastuzumab, added to a cisplatin/fluoropyrimidine chemotherapy doublet is now a standard treatment option for patients with HER-2 positive disease. Anti-HER-2 targeted agents are presently undergoing substantial clinical evaluation in advanced gastric cancer, with currently relatively limited evaluation in the perioperative and adjuvant settings. We are hopeful that further randomized studies will be undertaken to determine the value of anti-HER2 targeting in early disease and whether the survival benefits reported in early breast cancer can be replicated in operable gastric cancer. Further clinical evaluation of novel combinations of chemotherapy with trastuzumab, novel antibodies including pertuzumab, antibody-drug conjugates such as trastuzumab-DM1, and small molecule inhibitors of HER-2 must be expedited in molecularly selected patients with both advanced and operable disease to improve outcomes for patients with esophagogastric cancer.

\section{Acknowledgment}

Branislav Bystricky, Alicia Okines and David Cunningham acknowledge National Health Service funding from the National Institute for Health Research (NIHR) Biomedical Research Centre.

\section{Disclosure}

Branislav Bystricky and Alicia Okines have no relevant conflicts of interest.

David Cunningham has received research funding from Amgen, Roche, Merck Sharp and Dohme, sanofi-aventis, and Merck Serono, and has participated in uncompensated advisory boards for Roche and Amgen.

\section{References}

1. Ferlay J, Shin HR, Bray F, Forman D, Mathers C, Parkin DM. GLOBOCAN 2008. Cancer Incidence and Mortality Worldwide: IARC CancerBase No 10 2010. http://globocan.iarc.fr. Accessed August 3, 2011.

2. Jemal A, Bray F, Center MM, Ferlay J, Ward E, Forman D. Global cancer statistics. CA Cancer J Clin. 2011;61(2):69-90.

3. Kubo A, Corley DA. Body mass index and adenocarcinomas of the esophagus or gastric cardia: a systematic review and meta-analysis. Cancer Epidemiol Biomarkers Prev. 2006;15(5):872-878.

4. van Grieken NC, Weiss MM, Meijer GA, et al. Helicobacter pylorirelated and -non-related gastric cancers do not differ with respect to chromosomal aberrations. J Pathol. 2000;192(3):301-306.

5. Boers JE, Meeuwissen H, Methorst N. HER2 status in gastro-oesophageal adenocarcinomas assessed by two rabbit monoclonal antibodies (SP3 and 4B5) and two in situ hybridization methods (FISH and SISH). Histopathology. 2011;58(3):383-394.
6. Bang YJ, Van Cutsem E, Feyereislova A, et al. Trastuzumab in combination with chemotherapy versus chemotherapy alone for treatment of HER2-positive advanced gastric or gastro-oesophageal junction cancer (ToGA): a phase 3, open-label, randomised controlled trial. Lancet. 2010;376(9742):687-697.

7. Bozzetti C, Negri FV, Lagrasta CA, et al. Comparison of HER2 status in primary and paired metastatic sites of gastric carcinoma. Br J Cancer. 2011;104(9):1372-1376.

8. Sakai K, Mori S, Kawamoto T, et al. Expression of epidermal growth factor receptors on normal human gastric epithelia and gastric carcinomas. J Natl Cancer Inst. 1986;77(5):1047-1052.

9. Kasprzyk PG, Song SU, Di Fiore PP, King CR. Therapy of an animal model of human gastric cancer using a combination of antierbB-2 monoclonal antibodies. Cancer Res. 1992;52(10):2771-2776.

10. Matsui Y, Inomata M, Tojigamori M, Sonoda K, Shiraishi N, Kitano S. Suppression of tumor growth in human gastric cancer with HER2 overexpression by an anti-HER2 antibody in a murine model. Int J Oncol. 2005;27(3):681-685.

11. Gong SJ, Jin CJ, Rha SY, Chung HC. Growth inhibitory effects of trastuzumab and chemotherapeutic drugs in gastric cancer cell lines. Cancer Lett. 2004;214(2):215-224.

12. Bang Y, Chung $\mathrm{H}, \mathrm{Xu}$ J, et al. Pathological features of advanced gastric cancer (GC): relationship to human epidermal growth factor receptor 2 (HER2) positivity in the global screening programme of the ToGA trial. J Clin Oncol. 2009;27(Suppl 15):Abstr 4556.

13. Songun I, Putter H, Kranenbarg EM, Sasako M, van de Velde CJ. Surgical treatment of gastric cancer: 15-year follow-up results of the randomised nationwide Dutch D1D2 trial. Lancet Oncol. 2010;11(5):439-449.

14. Bonenkamp JJ, Songun I, Hermans J, et al. Randomised comparison of morbidity after D1 and D2 dissection for gastric cancer in 996 Dutch patients. Lancet. 1995;345(8952):745-748.

15. Sasako M, Sano T, Yamamoto S, et al. D2 lymphadenectomy alone or with para-aortic nodal dissection for gastric cancer. $N$ Engl J Med. 2008;359(5):453-462.

16. Macdonald JS, Smalley SR, Benedetti J, et al. Chemoradiotherapy after surgery compared with surgery alone for adenocarcinoma of the stomach or gastroesophageal junction. N Engl J Med. 2001;345(10):725-730.

17. Macdonald JS, Benedetti J, Smalley S, et al. Chemoradiation of resected gastric cancer: a 10-year follow-up of the phase III trial INT0116 (SWOG 9008). J Clin Oncol. 2009; p. suppl;abstr 4515.

18. Kwon HC, Kim MC, Kim KH, et al. Adjuvant chemoradiation versus chemotherapy in completely resected advanced gastric cancer with D2 nodal dissection. Asia Pac J Clin Oncol. 2010;6(4):278-285.

19. Dikken JL, Jansen EP, Cats A, et al. Impact of the extent of surgery and postoperative chemoradiotherapy on recurrence patterns in gastric cancer. J Clin Oncol. 2010;28(14):2430-2436.

20. Cunningham D, Allum WH, Stenning SP, et al. Perioperative chemotherapy versus surgery alone for resectable gastroesophageal cancer. N Engl J Med. 2006;355(1):11-20.

21. Ychou M, Boige V, Pignon JP, et al. Perioperative chemotherapy compared with surgery alone for resectable gastroesophageal Adenocarcinoma: A FNCLCC and FFCD multicenter Phase III trial. $J$ Clin Oncol. 2011;29(13):1715-1721.

22. Paoletti X, Oba K, Burzykowski T, et al. Benefit of adjuvant chemotherapy for resectable gastric cancer: a meta-analysis. JAMA. 2010; 303(17):1729-1737.

23. Sakuramoto S, Sasako M, Yamaguchi T, et al. Adjuvant chemotherapy for gastric cancer with S-1, an oral fluoropyrimidine. $N$ Engl J Med. 2007;357(18):1810-1820.

24. Sasako M, Kinoshita T, Furukawa H, et al; for ACTS-GC group Five-year results of the randomized phase III trial comparing S-1 monotherapy versus surgery alone for stage II/III gastric cancer patients after curative D2 gastrectomy (ACTS-GC study). Ann Oncol. 2010; 21(Suppl 8):viii225-viii49, Abst 709PD.

25. Cho HS, Mason K, Ramyar KX, et al. Structure of the extracellular region of HER2 alone and in complex with the Herceptin Fab. Nature. 2003;421(6924):756-760. 
26. Garrett TP, McKern NM, Lou M, et al. The crystal structure of a truncated ErbB2 ectodomain reveals an active conformation, poised to interact with other ErbB receptors. Mol Cell. 2003;11(2):495-505.

27. Pinkas-Kramarski R, Shelly M, Glathe S, Ratzkin BJ, Yarden Y. Neu differentiation factor/neuregulin isoforms activate distinct receptor combinations. J Biol Chem. 1996;271(32):19029-19032.

28. Worthylake R, Opresko LK, Wiley HS. ErbB-2 amplification inhibits down-regulation and induces constitutive activation of both ErbB-2 and epidermal growth factor receptors. J Biol Chem. 1999; 274(13):8865-8874.

29. Hendriks BS, Orr G, Wells A, Wiley HS, Lauffenburger DA. Parsing ERK activation reveals quantitatively equivalent contributions from epidermal growth factor receptor and HER2 in human mammary epithelial cells. J Biol Chem. 2005;280(7):6157-6169.

30. Spector NL, Blackwell KL. Understanding the mechanisms behind trastuzumab therapy for human epidermal growth factor receptor 2-positive breast cancer. J Clin Oncol. 2009;27(34):5838-5847.

31. Carter P, Presta L, Gorman CM, et al. Humanization of an antip185HER2 antibody for human cancer therapy. Proc Natl Acad Sci U S A. 1992;89(10):4285-4289.

32. Kim SY, Kim HP, Kim YJ, et al. Trastuzumab inhibits the growth of human gastric cancer cell lines with HER2 amplification synergistically with cisplatin. Int J Oncol. 2008;32(1):89-95.

33. Fujimoto-Ouchi K, Sekiguchi F, Yasuno H, Moriya Y, Mori K, Tanaka Y. Antitumor activity of trastuzumab in combination with chemotherapy in human gastric cancer xenograft models. Cancer Chemother Pharmacol. 2007;59(6):795-805.

34. Yonemura Y, Ninomiya I, Yamaguchi A, et al. Evaluation of immunoreactivity for erbB-2 protein as a marker of poor short term prognosis in gastric cancer. Cancer Res. 1991;51(3):1034-1108.

35. Grabsch H, Sivakumar S, Gray S, Gabbert HE, Muller W. HER2 expression in gastric cancer: rare, heterogeneous and of no prognostic value - conclusions from 924 cases of two independent series. Cell Oncol. 2010;32(1-2):57-65.

36. Hofmann M, Stoss O, Shi D, et al. Assessment of a HER2 scoring system for gastric cancer: results from a validation study. Histopathology. 2008;52(7):797-805.

37. Ruschoff J, Dietel M, Baretton G, et al. HER2 diagnostics in gastric cancer-guideline validation and development of standardized immunohistochemical testing. Virchows Arch. 2010;457(3):299-307.

38. Wolff AC, Hammond ME, Schwartz JN, et al. American Society of Clinical Oncology/College of American Pathologists guideline recommendations for human epidermal growth factor receptor 2 testing in breast cancer. J Clin Oncol. 2007;25(1):118-145.

39. Joensuu H, Bono P, Kataja V, et al. Fluorouracil, epirubicin, and cyclophosphamide with either docetaxel or vinorelbine, with or without trastuzumab, as adjuvant treatments of breast cancer: final results of the FinHer Trial. J Clin Oncol. 2009;27(34):5685-5692.

40. Romond EH, Perez EA, Bryant J, et al. Trastuzumab plus adjuvant chemotherapy for operable HER2-positive breast cancer. NEngl J Med. 2005;353(16):1673-1684.

41. Slamon D, Eiermann W, Robert N, et al. Phase III Randomized Trial Comparing Doxorubicin and Cyclophosphamide Followed by Docetaxel $(\mathrm{AC}->\mathrm{T})$ with Doxorubicin and Cyclophosphamide Followed by Docetaxel and Trastuzumab (AC - $>$ TH) with Docetaxel, Carboplatin and Trastuzumab (TCH) in Her2neu Positive Early Breast Cancer Patients: BCIRG 006 Study. Cancer Res. 2009;69(Suppl 24):Abstract 62

42. Slamon DJ, Leyland-Jones B, Shak S, et al. Use of chemotherapy plus a monoclonal antibody against HER2 for metastatic breast cancer that overexpresses HER2. N Engl J Med. 2001;344(11):783-792.

43. Spielmann M, Roche H, Delozier T, et al. Trastuzumab for patients with axillary-node-positive breast cancer: results of the FNCLCC-PACS 04 trial. J Clin Oncol. 2009;27(36):6129-6134.

44. Gianni L, Dafni U, Gelber RD, et al. Treatment with trastuzumab for 1 year after adjuvant chemotherapy in patients with HER2-positive early breast cancer: a 4-year follow-up of a randomised controlled trial. Lancet Oncol. 2011;12(3):236-244.
45. Buzdar AU, Ibrahim NK, Francis D, et al. Significantly higher pathologic complete remission rate after neoadjuvant therapy with trastuzumab, paclitaxel, and epirubicin chemotherapy: results of a randomized trial in human epidermal growth factor receptor 2-positive operable breast cancer. J Clin Oncol. 2005;23(16):3676-3685.

46. Untch M, Rezai M, Loibl S, et al. Neoadjuvant treatment with trastuzumab in HER2-positive breast cancer: results from the GeparQuattro study. J Clin Oncol. 2010;28(12):2024-2031.

47. Untch M, Muscholl M, Tjulandin S, et al. First-line trastuzumab plus epirubicin and cyclophosphamide therapy in patients with human epidermal growth factor receptor 2-positive metastatic breast cancer: cardiac safety and efficacy data from the Herceptin, Cyclophosphamide, and Epirubicin (HERCULES) trial. J Clin Oncol. 2010;28(9): 1473-1480.

48. Gianni L, Eiermann W, Semiglazov V, et al. Neoadjuvant chemotherapy with trastuzumab followed by adjuvant trastuzumab versus neoadjuvant chemotherapy alone, in patients with HER2-positive locally advanced breast cancer (the NOAH trial): a randomised controlled superiority trial with a parallel HER2-negative cohort. Lancet. 2010; 375(9712):377-384.

49. Blazeby JM, Vickery CW. Quality of life in patients with cancers of the upper gastrointestinal tract. Expert Rev Anticancer Ther. 2001;1(2): 269-276.

50. Satoh T, Leon J, Lopez RI, et al. Quality of life results from a phase III trial of trastuzumab plus chemotherapy in first-line HER2-positive advanced gastric and GE junction cancer. 2010 Gastrointestinal Cancers Symposium. Orlando, Florida, USA; 2010:Abst 7.

51. Smith I, Procter M, Gelber RD, et al. 2-year follow-up of trastuzumab after adjuvant chemotherapy in HER2-positive breast cancer: a randomised controlled trial. Lancet. 2007;369(9555):29-36.

52. Allegra CJ, Yothers G, O’Connell MJ, et al. Phase III trial assessing bevacizumab in stages II and III carcinoma of the colon: results of NSABP protocol C-08. J Clin Oncol. 2011;29(1):11-16.

53. DeGramont A, Van Cutsem E, Tabernero J, et al. AVANT: results from a randomized, three-arm multinational phase III study to investigate bevacizumab with either XELOX or FOLFOX4 versus FOLFOX4 alone as adjuvant treatment for colon cancer. J Clin Oncol. 2011; 29(Suppl 4):Abstr 362.

54. Huang J, Sargent DJ, Mahoney MR, et al. Adjuvant FOLFIRI with or without cetuximab in patients with resected stage III colon cancer: NCCTG Intergroup phase III trial N0147. J Clin Oncol. 2011; 29(Suppl 4):Abstr 363.

55. NCT01130337. A Study of Capecitabine [Xeloda] in Combination With Trastuzumab [Herceptin] and Oxaliplatine in Patients With Resectable Gastric Cancer. 2010 [cited 2011 13.4]. Available from: http://clinicaltrials.gov/ct2/show/NCT01130337.

56. RTOG-1010. Radiation Therapy, Paclitaxel, and Carboplatin With or Without Trastuzumab in Treating Patients With Esophageal Cancer. 2010 [cited 2011 13.4]. Available from: http://clinicaltrials.gov/ct2/ show/NCT01196390.

57. Baselga J, Gelmon KA, Verma S, et al. Phase II trial of pertuzumab and trastuzumab in patients with human epidermal growth factor receptor 2-positive metastatic breast cancer that progressed during prior trastuzumab therapy. J Clin Oncol. 2010;28(7):1138-1144.

58. Makhija S, Amler LC, Glenn D, et al. Clinical activity of gemcitabine plus pertuzumab in platinum-resistant ovarian cancer, fallopian tube cancer, or primary peritoneal cancer. J Clin Oncol. 2010;28(7): 1215-1223.

59. Burris HA 3rd, Rugo HS, Vukelja SJ, et al. Phase II study of the antibody drug conjugate trastuzumab-DM1 for the treatment of human epidermal growth factor receptor 2 (HER2)-positive breast cancer after prior HER2-directed therapy. J Clin Oncol. 2011;29(4):398-405.

60. NCT00829166. An Open-Label Study of Trastuzumab-MCC-DM1 (T-DM1) vs Capecitabine + Lapatinib in Patients With HER2-Positive Locally Advanced or Metastatic Breast Cancer (EMILIA). 2009 [cited 2011 13.4]. Available from: http://clinicaltrials.gov/ct2/show/ NCT00829166. 
61. NCT01120184. A Study of Trastuzumab-DM1 Plus Pertuzumab Versus Trastuzumab [Herceptin] Plus a Taxane in Patients With Metastatic Breast Cancer (MARIANNE). 2010 [cited 2011 13.4]. Available from: http://clinicaltrials.gov/ct2/show/NCT01120184.

62. Barok M, Tanner M, Koninki K, Isola J. Trastuzumab-DM1 is highly effective in preclinical models of HER2-positive gastric cancer. Cancer Lett. 2011;28(2):171-179.

63. Geyer CE, Forster J, Lindquist D, et al. Lapatinib plus capecitabine for HER2-positive advanced breast cancer. $N$ Engl J Med. 2006; 355(26):2733-2743.

64. Iqbal S, Goldman B, Fenoglio-Preiser CM, et al. Southwest Oncology Group study S0413: a phase II trial of lapatinib (GW572016) as first-line therapy in patients with advanced or metastatic gastric cancer. Ann Oncol. 2011 Mar 17. [Epub ahead of print, doi:0.1093/annonc/mdr021].

65. NCT00486954. Lapatinib in Combination With Weekly Paclitaxel in Patients With ErbB2 Amplified Advanced Gastric Cancer. 2007 [cited 2011 13.4]. Available from: http://clinicaltrials.gov/ct2/show/ NCT00486954.

66. NCT00680901. LOGiC - Lapatinib Optimization Study in ErbB2 (HER2) Positive Gastric Cancer: A Phase III Global, Blinded Study Designed to Evaluate Clinical Endpoints and Safety of Chemotherapy Plus Lapatinib. 2008 [cited 2011 13.4]. Available from: http:// clinicaltrials.gov/ct2/show/NCT00680901.

67. Satoh T, Bang Y, Wang J, et al. Interim safety analysis from TYTAN: a phase III Asian study of lapatinib in combination with paclitaxel as second-line therapy in gastric cancer. J Clin Oncol. 2010;28 (Suppl 15):Abstr 4057.

68. Hecht J, Bang Y, Sobrero A, et al. A phase III study of CapeOx +/- lapatinib in HER2 positive locally-advanced/metastatic upper gastrointestinal adenocarcinoma: interim safety results. Eur J Cancer Suppl. 2009;7(2):385.

69. Gonzales AJ, Hook KE, Althaus IW, et al. Antitumor activity and pharmacokinetic properties of PF-00299804, a second-generation irreversible pan-erbB receptor tyrosine kinase inhibitor. Mol Cancer Ther. 2008;7(7):1880-1889.

70. Campbell A, Reckamp KL, Camidge DR, et al. PF-00299804 (PF299) patient (pt)-reported outcomes (PROs) and efficacy in adenocarcinoma (adeno) and nonadeno non-small cell lung cancer (NSCLC): a phase (P) II trial in advanced NSCLC after failure of chemotherapy (CT) and erlotinib (E). J Clin Oncol. 2010;28(Suppl 15):Abstr 7596.

71. NCT01152853. PF-00299804 Monotherapy in Patients With HER-2 Positive Advance Gastric Cancer (PF299804-AGC). 2010 [cited 2011 13.4]; Available from: http://clinicaltrials.gov/ct2/show/NCT01152853.

72. Swaby RF, Blackwell K, Jiang Z, et al. Neratinib in combination with trastuzumab for the treatment of advanced breast cancer: a phase 1/2 study. 2009 Breast Cancer Symposium. San Francisco, California, USA; 2009:Abstr 243.

73. Hickinson DM, Klinowska T, Speake G, et al. AZD8931, an equipotent, reversible inhibitor of signaling by epidermal growth factor receptor, ERBB2 (HER2), and ERBB3: a unique agent for simultaneous ERBB receptor blockade in cancer. Clin Cancer Res. 2010;16(4):1159-1169.

74. Buzdar AU, Valero V, Ibrahim NK, et al. Neoadjuvant therapy with paclitaxel followed by 5 -fluorouracil, epirubicin, and cyclophosphamide chemotherapy and concurrent trastuzumab in human epidermal growth factor receptor 2-positive operable breast cancer: an update of the initial randomized study population and data of additional patients treated with the same regimen. Clin Cancer Res. 2007;13(1):228-233.

Gastrointestinal Cancer: Targets and Therapy

\section{Publish your work in this journal}

Gastrointestinal Cancer: Targets and Therapy is an international, peer-reviewed, open access journal focusing on gastro-intestinal cancer research, identification of therapeutic targets and the optimal use of preventative and integrated treatment interventions to achieve improved outcomes, enhanced survival and quality of life for the
75. Jain KK, Casper ES, Geller NL, et al. A prospective randomized comparison of epirubicin and doxorubicin in patients with advanced breast cancer. J Clin Oncol. 1985;3(6):818-826.

76. von Minckwitz G, du Bois A, Schmidt M, et al. Trastuzumab beyond progression in human epidermal growth factor receptor 2-positive advanced breast cancer: a german breast group 26/breast international group 03-05 study. J Clin Oncol. 2009;27(12):1999-2006.

77. Shimoyama R, Yasui H, Boku N, et al. Weekly paclitaxel for heavily treated advanced or recurrent gastric cancer refractory to fluorouracil, irinotecan, and cisplatin. Gastric Cancer. 2009;12(4):206-211.

78. Thuss-Patience PC, Kretzschmar A, Deist T, et al. Irinotecan versus best supportive care (BSC) as second-line therapy in gastric cancer: a randomized phase III study of the Arbeitsgemeinschaft Internistische Onkologie (AIO). J Clin Oncol. 2009;27(Suppl 15):Abstr 4540.

79. Cunningham D, Park S, Kang Y, et al. Randomized phase II study of PEP02, irinotecan, or docetaxel as a second-line therapy in gastric or gastroesophageal junction adenocarcinoma. J Clin Oncol. 2011;29(Suppl 4):Abstr 6.

80. Wainberg ZA, Anghel A, Desai AJ, et al. Lapatinib, a dual EGFR and HER2 kinase inhibitor, selectively inhibits HER2-amplified human gastric cancer cells and is synergistic with trastuzumab in vitro and in vivo. Clin Cancer Res. 2010;16(5):1509-1519.

81. Arpino G, Gutierrez C, Weiss H, et al. Treatment of human epidermal growth factor receptor 2-overexpressing breast cancer xenografts with multiagent HER-targeted therapy. J Natl Cancer Inst. 2007;99(9):694-705.

82. Janne PA, Boss DS, Camidge DR, et al. Phase I dose-escalation study of the pan-HER inhibitor, PF299804, in patients with advanced malignant solid tumors. Clin Cancer Res. 2011;17(5):1131-1139.

83. Eichhorn PJ, Gili M, Scaltriti M, et al. Phosphatidylinositol 3-kinase hyperactivation results in lapatinib resistance that is reversed by the mTOR/phosphatidylinositol 3-kinase inhibitor NVP-BEZ235. Cancer Res. 2008;68(22):9221-9230.

84. Brachmann SM, Hofmann I, Schnell C, et al. Specific apoptosis induction by the dual PI3 K/mTor inhibitor NVP-BEZ235 in HER2 amplified and PIK3CA mutant breast cancer cells. Proc Natl Acad Sci U S A. 2009;106(52):22299-22304.

85. NCT01145404. Trial of Lapatinib Versus Lapatinib With Capecitabine in Her2+ Metastatic Gastro-Esophageal Cancer (GastroLap). 2010 [cited 2011 13.4]. Available from: http://clinicaltrials.gov/ct2/show/ NCT01145404.

86. NCT00526669. Study For Patients With Untreated Gastric Cancer Who Will Receive Capecitabine And Lapatinib. 2007 [cited 2011 13.4]. Available from: http://clinicaltrials.gov/ct2/show/NCT00526669.

87. NCT01123473. Epirubicin Hydrochloride, Cisplatin, and Fluorouracil or Capecitabine With or Without Lapatinib Ditosylate as First-Line Therapy in Treating Patients With Stomach Cancer or Gastroesophageal Junction Cancer. 2010 [cited 2011 13.4]. Available from: http:// clinicaltrials.gov/ct2/show/NCT01123473.

88. NCT00838539. Study Evaluating Neratinib In Combination With Temsirolimus In Subjects With Solid Tumors. 2009 [cited 2011 13.4]. Available from: http://clinicaltrials.gov/ct2/show/NCT00838539.

89. NCT01003158. Study Assessing Safety and Tolerability of AZD8931 Alone or in Combination With Paclitaxel in Japanese Patients. 2009 [cited 2011 13.4]. Available from: http://clinicaltrials. gov/ct2/show/NCT01003158.

cancer patient. The manuscript management system is completely online and includes a very quick and fair peer-review system. Visit $\mathrm{http}: / / \mathrm{www}$.dovepress.com/testimonials.php to read real quotes from published authors. 